\title{
Cardiac Resynchronization Therapy Induces Adaptive Metabolic Transitions in the Metabolomic Profile of Heart Failure
}

\author{
Emirhan Nemutlu, PhD, Song Zhang, PhD, Yi-Zhou Xu, MD, Andre Terzic, MD, PhD, Li \\ Zhong, MD, Petras D. Dzeja, PhD, and Yong-Mei Cha, MD \\ Division of Cardiovascular Diseases, Departments of Medicine (E.M., Y-Z.X, S.Z., A.T., L.Z., \\ P.D., Y-M.C.) and Molecular Pharmacology and Experimental Therapeutics (E.M., S.Z., \\ A.T.,P.D.), Mayo Clinic, Rochester, Minnesota and Department of Analytical Chemistry, Faculty of \\ Pharmacy, University of Hacettepe, 06100 Ankara, Turkey (E.M.)
}

\begin{abstract}
Background-Heart failure (HF) is associated with ventricular dyssynchrony and energetic inefficiency, which can be alleviated by cardiac resynchronization therapy (CRT). The aim of this study was to determine the metabolomic signature in $\mathrm{HF}$ and its prognostic value for the response to CRT.
\end{abstract}

Methods-This prospective study consisted of 24 patients undergoing CRT for advanced HF and 10 control patients who underwent catheter ablation for supraventricular arrhythmia but not CRT. Blood samples were collected before and 3 months after CRT. Metabolomic profiling of plasma samples was performed using gas chromatography-mass spectrometry and nuclear magnetic resonance.

Results-The plasma metabolomic profile was altered in the HF patients, with a distinct panel of metabolites, including Krebs cycle and lipid, amino acid, and nucleotide metabolism. CRT improved the metabolic profile. The succinate/glutamate ratio, an index of Krebs cycle activity, improved from $0.58 \pm 0.13$ to $2.84 \pm 0.60(P<.05)$. The glucose/palmitate ratio, an indicator of the balance between glycolytic and fatty acid metabolism, increased from $0.96 \pm 0.05$ to $1.54 \pm 0.09$ $(P<.01)$. Compared with the nonresponders to CRT, the responders had a distinct baseline plasma metabolomic profile, including higher isoleucine, phenylalanine, leucine, glucose, and valine levels and lower glutamate levels at baseline $(P<.05)$.

Conclusion-CRT improves plasma metabolomic profile of HF patients indicating harmonization of myocardial energy substrate metabolism. CRT responders may have a favorable metabolic profile as a potential biomarker for predicting CRT outcome.

\section{Keywords}

heart failure; metabolism; cardiac resynchronization therapy

Correspondence to Yong-Mei Cha, MD and Petras Dzeja, PhD, Division of Cardiovascular Diseases, Mayo Clinic, 200 First Street SW, Rochester, MN 55905. ycha@mayo.edu and dzeja.petras@mayo.edu.

Disclosures

None. 


\section{Introduction}

Heart failure (HF) is a complex clinical syndrome associated with cardiac structural, metabolic, and functional deficiency ${ }^{1-4}$. Cardiac remodeling in HF involves ventricular electrical and mechanical dyssynchrony, which results in metabolic heterogeneity and energy insufficiency ${ }^{5-7}$. Impaired myocardial substrate metabolism and phosphotransfer dynamics further contribute to HF abnormalities ${ }^{5,6,8-11}$. Cardiac resynchronization therapy (CRT) effectively treats HF by correcting cardiac dyssynchrony, resulting in improvement of HF symptoms, ventricular function, quality of life, and overall survival ${ }^{12,13}$. The reduction of mechanical dyssynchrony within the left ventricle and subsequent improvement in left ventricular (LV) efficiency and oxygen consumption have been considered the main mechanistic benefits of $\mathrm{CRT}^{14,15}$. Although the subcellular metabolic and energy systems are affected in $\mathrm{HF}^{16,17}$, little is known about the effect of CRT on myocardial substrate metabolism $^{18,19}$.

Metabolomics analyzes a large range of metabolites from multiple metabolic pathways and increasingly demonstrates the potential to provide individualized and predictive information for disease progression and personalization of treatment ${ }^{20-31}$. Determining the association of metabolomic changes with HF and CRT is necessary for understanding the mechanisms of re-regularization of cardiac metabolism derived from this therapy ${ }^{18}$. The aim of this study was to determine 1) the metabolomic signature in HF and the therapeutic effects of CRT on the metabolomic profile and the pathways of adaptive metabolic remodeling that lead to recovery of myocardial function and 2) the panel of metabolic markers that predicts which patients will respond to CRT.

\section{Methods}

\section{Study Patients}

This single-center prospective study consisted of 24 consecutive patients who received CRT-D (defibrillation) for advanced HF at Mayo Clinic, Rochester, Minnesota, from March 1 through November 30, 2010. All patients were clinically recommended for device implantation according to current guidelines for $\mathrm{CRT}^{32}$. The Mayo Clinic Institutional Review Board approved this study, and all patients provided signed consent for the study. Ten age-matched control patients underwent catheter ablation for supraventricular arrhythmia with a normal left ventricular ejection fraction (LVEF) of greater than $55 \%$.

\section{Baseline Evaluation}

All HF patients underwent a baseline evaluation before CRT, including assessment of New York Heart Association (NYHA) functional class, concomitant cardiovascular conditions (e.g., hypertension, coronary artery disease, and diabetes mellitus), electrocardiographic QRS duration and morphologic characteristics, and transthoracic echocardiography. Echocardiographic parameters included LV end-systolic and diastolic dimensions, LVEF, and pulmonary artery systolic pressure. Medication use was recorded and confirmed that patients were taking optimal medication dose for HF. Patients continued on stable medication dosage during the study. 


\section{CRT Implantation and Blood Sample Collection}

All patients underwent conscious sedation throughout the procedure for CRT implantation. The right femoral artery was cannulated for continuous monitoring during the procedure in both HF and control patients. Blood samples were taken from a peripheral vein before the device was implanted. These blood samples $(5 \mathrm{~mL})$ were collected into EDTA tubes containing $100 \mu \mathrm{L}$ of injected stop solution (dipyrimidole, $10 \mu \mathrm{M}$; EHNA, $5 \mu \mathrm{M}$; iodotubercidin, $2 \mu \mathrm{M}$; AOPCP, $50 \mu \mathrm{M}$, in $0.9 \% \mathrm{NaCl}$ ) to reduce blood nucleotide metabolism; stored on ice; and then centrifuged at $3,200 \mathrm{rpm}$ for 10 minutes at $4^{\circ} \mathrm{C}$. Plasma aliquots were stored at $-80^{\circ} \mathrm{C}$ until analyses.

\section{Patient Follow-up}

The HF patients returned for a clinical follow-up 3 months after CRT. The NYHA functional class was reassessed, and echocardiography was repeated. Peripheral venous blood samples were collected for post-CRT metabolic measurements. Metabolomic profile was successfully determined in 19 patients. Improvement in LVEF by more than 5\% and reduction of NYHA $\geq 1$ class were considered as CRT responders.

\section{Metabolomic Analyses}

\section{Sample Treatment and Instrumental Conditions for GC-MS Metabolomic} Analysis-For gas chromatography-mass spectrometry (GC-MS), plasma $(100 \mu \mathrm{L})$ was extracted using a $900-\mu \mathrm{L}$ methanol:water $(8: 1, \mathrm{v} / \mathrm{v})$ mixture containing $5 \mu \mathrm{g}$ internal standard, myristic- $\mathrm{d}_{27}$ acid, at ambient temperature ${ }^{33}$. Supernatant $(900 \mu \mathrm{L})$ was transferred and completely dried in a vacuum concentrator. Subsequently, the tubes were methoximated and derivatized and then analyzed using an Agilent 6890 GC oven with Agilent 5973 MS $^{34}$.

\section{Sample Treatment and Instrumental Conditions for ${ }^{1} \mathrm{H}$ NMR Metabolomic} Analysis-For nuclear magnetic resonance (NMR) imaging, plasma ( $60 \mu \mathrm{L})$ was diluted $140 \mu \mathrm{L}$ with $0.2 \mathrm{M}$ phosphate buffer $(\mathrm{pH} 7.4): \mathrm{D}_{2} \mathrm{O}$ containing a mixture of $16 \mathrm{mM}$ formate and $4 \mathrm{mM}$ TSP $(1: 1, \mathrm{v} / \mathrm{v})$. After filtering $(0.22 \mu \mathrm{m})$, the samples were transferred into a 3mm-diameter NMR tube. High-resolution ${ }^{1} \mathrm{H}$ NMR spectra were acquired at $600 \mathrm{MHz}$ on a Bruker Avance III 600 spectrometer. Spectra peaks were identified according to Chenomx NMR Suite 6.1 software and data in the literature ${ }^{35,36}$.

Metabolomic Data Analysis-The data files from GC-MS analyses were deconvoluted using AMDIS software ${ }^{37}$, and then SpectConnect (http://spectconnect.mit.edu/index.php) was used to list and track metabolite peaks ${ }^{38}$. Quality control-based signal correction and integration of data were used to minimize intra- and inter-day variations ${ }^{29}$. The Agilent Fiehn GC/MS Metabolomics RTL Library was used for metabolite identification. Data analysis of ${ }^{1} \mathrm{H}$ NMR spectra was done with the MestRenova (Mestrelab). The identified peaks were integrated and normalized according to an internal standard (fumarate). Analysis of the ${ }^{1} \mathrm{H}$ NMR spectra and GC-MS chromatogram permitted detection over 400 metabolite peaks, major of which are presented in Supplemental Table 1. Orthogonal partial least squares discriminant analysis (OPLS-DA) was performed using Umetrics SIMCA-P+ version 12.0. The variable importance in the projection values was calculated to identify a 
panel of the most important metabolites, and regression coefficients were used to validate group separation.

\section{Statistical Analysis}

All data are expressed as mean \pm standard error of the mean. The Student unpaired $t$ test was used, and differences were considered statistically significant at $P<.05$. One-way analysis of variance was used to test differences among groups.

\section{Results}

\section{Baseline Characteristics}

The baseline demographic characteristics in 24 CRT patients and 10 control patients are shown in Table 1. Age and sex were not significantly different between the 2 groups. Ten HF patients (42\%) had ischemic cardiomyopathy. The incidence of hypertension and diabetes was not significantly different in the 2 groups. All HF patients had NYHA class III symptoms. The mean LVEF was $23.5 \pm 6.3 \%$ in the CRT group compared with $62.9 \% \pm 3.3 \%$ in the control group $(P<.001)$. More patients in the CRT group received angiotensinconverting enzyme inhibitors or angiotensin receptor blockers $(P<.001)$ and statins $(P=.002)$ than in the control group.

\section{Improvement in HF After CRT}

At 3-month follow-up, the post-CRT group had significant improvement in HF symptoms. The severity of the NYHA class was reduced, while LV systolic function, the severity of mitral regurgitation, and elevated pulmonary artery systolic pressure were improved (Table 2). LVEF improved from $23.5 \% \pm 6.3 \%$ to $32.0 \pm 8.6 \%(P<.001)$.

\section{Shift in Plasma Metabolomic Profile Associated With HF}

Here, using metabolomic technologies we demonstrate that the HF pre-CRT group had a significantly altered plasma metabolomic profile compared with the control group (Figure 1A). The integral metabolomic profile in the HF pre-CRT group clustered separately from the control group on the OPLS-DA score plot. The panel of most important metabolites in group discrimination is shown in Figure 1B. The full list of major metabolites detected in the HF pre- and post-CRT and control groups is presented in Supplemental Table 1. In HF pre-CRT, as markers of altered mitochondrial function, succinate was reduced and glutamate was increased. A decrease in amino and fatty acids and an increase in urate levels were apparent (Figure 1C). Metabolic pathways most affected in HF patients included amino acid and lipid metabolism and Krebs cycle, with a smaller contribution of carbohydrate metabolism, energy transfer, neurotransmitter metabolism, ammonia detoxification, and nucleotide degradation metabolism (Figure 1D).

\section{Changes in Plasma Metabolomic Profile After CRT}

Three months after CRT, the plasma metabolomic profile was significantly changed (Figure 2A). Scores of the integral metabolomic profile of each HF patient post-CRT clustered separately from the original state on the OPLS-DA plot, indicating a transition to a new 
metabolic state. Moreover, changes in metabolomic profile specific to mechanical and electrical dyssynchrony can be evaluated by comparing HF pre-CRT and post-CRT (Figure 2). An integrated panel of the most important metabolites allowing post-CRT group discrimination included the branched-chain amino acid isoleucine, glutamine and glycerol-1-phosphate associated with mitochondrial oxidation, and metabolites of fatty acid and amino acid metabolism (Figure 2B). The pattern of metabolite changes indicates improved substrate metabolism after CRT (Figure 2C). Among the most improved metabolic pathways were amino acid and lipid metabolism and Krebs cycle, with a reduced contribution of substrate shuttling, energy/oxidative stress, ammonia detoxification, and myofibrillar protein turnover (Figure 2D). Several metabolites including cysteine, glutamate, glycerol-1-phosphate, glycine, malate and tryptophan restored to control values after CRT (Supplemental Table 1). This pattern is supported by changes in the succinate/glutamate ratio, an index of Krebs cycle activity ${ }^{39}$, which improved from $0.58 \pm 0.13$ to $2.84 \pm 0.60$ ( $P<$. 05) after CRT (Figure 3A). Similarly, the citrate/glutamate ratio increased from $0.69 \pm 0.12$ to $2.62 \pm 0.63(P<.001)$ after CRT. The glucose/palmitate ratio, an indicator of the balance between glycolytic and fatty acid metabolism, increased from $0.96 \pm 0.05$ to $1.54 \pm 0.09$ ( $P<$. 001). The glutamine/glutamate ratio, an indicator of ammonia fixation, improved from $0.66 \pm 0.12$ to $2.71 \pm 0.46(P<.001)$ after CRT. Although HF post-CRT metabolomic profile improved significantly, it was still different from the control group (Supplemental Figure 1A). A panel of the most important metabolites in group distinction included glutamic and succinic acids, glycerol, glycerol-1-phosphate, free fatty and amino acids (Supplemental Figure 1B), reflecting Krebs cycle, lipid and amino acid metabolism.

\section{Correlation Between Plasma Metabolites and Cardiac Performance}

A panel of representative metabolites correlated with LVEF at baseline in HF pre-CRT group (Figure 3B). Plasma levels of glutamate and urate, glucose, threitol, urea, and creatinine correlated negatively, while levels of linoleate, cysteine, glycine, serine, glycerol, serotonin, threonine, and succinate correlated positively with LVEF in HF pre-CRT group. Three months after CRT plasma levels of glucose, serotonin, lactate, alanine and glycerol-1phosphate correlated positively with LVEF (Figure 3C). Glucose levels positively correlated with delta LVEF improvement, while myristic acid negative correlation with delta LVEF (Figure 3D). Transitions in the global metabolomic matrix were compared between control, HF pre-CRT and post-CRT patient groups (Supplemental Figure 2). In the heat map, the red color indicates an increased metabolite level, and green indicates a decreased metabolite level. The HF post-CRT group had a distinct cluster of decreased metabolite levels and another cluster with increased metabolite levels. The cluster of decreased metabolites included glycerol, $N$-methylhistidine, myristic, oleic, palmitic, stearic, palmitoleic, heptadecanoic, tryptophan, glycerol-1-phosphate, gluconate, 2-hydoxybutyrate (2-HB), and malate. The cluster of increased metabolites included amino acids proline, leucine, and glutamine, as well as acetate, succinate, glycolic acid, acetoacetate, 3-hydoxybutyrate (3HB), and others (Supplemental Figure 2).

\section{Baseline Metabolomic Profile Predicts Improvement in LVEF After CRT}

Of $24 \mathrm{HF}$ patients who received CRT, 10 (41\%) had an improvement in LVEF by more than $5 \%$ and reduction of NYHA $\geq 1$ class and were considered CRT responders. This group of 
patients differed in their pre-CRT baseline plasma metabolomic profile from nonresponders in the OPLS-DA plot analysis (Figure 4A). Panel of most important metabolites allowing distinction between responders and non-responders to CRT therapy included isoleucine, phenylalanine, leucine, glutamate, acetone, phosphoric acid, glycerol, lactate, valine, glucose and other metabolites (Figure 4B). Among them were three branched-chain amino acids - isoleucine, leucine and valine, essential for protein synthesis and metabolic signaling, with lower levels in non-responders (Figure 4C). Patients who responded to CRT had higher amino acids phenylalanine, isoleucine, leucine, valine, and glucose and lower glutamate levels (Figure 4C; $P<.05$ ).

By comparing pre- and post-CRT metabolite values, responders had higher 2hydroxypyridine, alanine, acetate, glycerol -1-P, isoleucine, N-methylhistidine, ornithine, oxalic acid, phosphoric acid, succinate and tyrosine levels 3 month after CRT (Supplemental Table 2), while levels of benzoic acid, galacturonic acid, gluconic acid, glutamic acid, and other metabolites were lower post- CRT. Non-responders had a different increased or reduced metabolites profile after CRT (Supplemental Table 2). Analyses of specific metabolic traits following CRT (Supplemental Table 3) reveal that nonresponders had significantly smaller and negative change in ornithine and higher delta in porphine levels.

\section{Discussion}

\section{Metabolomic Signatures in HF}

Systolic HF is associated with cardiac conduction abnormalities and dyssynchrony, which results in poorly coordinated contractions precipitating energy inefficiency and LV dysfunction 1, 5, 6, 13, 40. Cardiac dyssynchrony affects many systems ranging from gene expression, ion channels, sarcomeres, mitochondrial energy metabolism and metabolic pathways ${ }^{41-43}$. CRT improves chamber mechano-energy efficiency, reducing morbidity and mortality of HF patients ${ }^{13-15,44}$. Emerging metabolomic profiling of disease phenotypes permits comprehensive and simultaneous systematic fingerprinting of multiple metabolites and metabolic pathways ${ }^{22,24-28}$. Here, metabolomic profiling indicates that HF patients have altered plasma metabolomic profiles, with a distinct panel of representative metabolites of lipid, amino acid, and nucleotide metabolism and mitochondrial Krebs cycle-associated substrates such as succinate and glutamate. Most altered in HF patients were the reduced level of succinate and the increased level of glutamate, the indicators of mitochondrial dysfunction and metabolic remodeling ${ }^{45}$. The lower succinate level suggests decreased concentration of Krebs cycle intermediates, which are critical for burning fats and carbohydrates ${ }^{45}, 46$. Diminished substrate metabolism is also indicated by changes in the succinate/glutamate and citrate/glutamate ratios, which were significantly decreased in HF. Thus, insufficient mitochondrial substrate utilization and metabolomic heterogeneity may contribute to energy deficiency in HF. The higher plasma levels of glutamate, urate, urea, and creatinine confer the alterations in Krebs cycle, amino acid changes, nucleotide degradation, and ammonia detoxification, the indicators of heart dysfunction ${ }^{29,47}$. Since glutamate and succinate are on opposite ends of the correlation panel, their plasma ratio could have a diagnostic and prognostic value. Thus, the plasma metabolomic fingerprint reflects an altered energy and metabolic environment in patients with HF. 
In HF, deficient substrate oxidation may cause a decrease in the ATP level and an increase in the end product of purine metabolism, such as urate levels ${ }^{22}$. This is a sign of an impairment of oxidative metabolism and highly correlates with measures of functional capacity and disease severity of $\mathrm{HF}^{1,5}$. Therefore, urate has been suggested as a marker of $\mathrm{HF}^{29}, 47$. We had similar findings in our study. The decreased levels of threonine, glycine, serine, and cysteine indicate energy deficiency and mitochondrial dysfunction in $\mathrm{HF}^{48}$. The plasma levels of glycerol, linoleate, myristate, and cholesterol, which were reduced in HF patients, may indicate reduced lipid metabolism. Similarly others using different analytical platforms and patient groups found altered metabolomic profiles in heart failure and identified candidate disease biomarkers ${ }^{19,29,49,50}$. Consistent with derangement in different metabolic pathways at a systemic level, as we observed in our study, HF patients had higher serum concentrations of phenylalanine, tyrosine, isoleucine, creatine, and lower serum levels of lactate, citrate and lysine ${ }^{49}$. In patients with heart failure significant changes in serum metabolomics profiles, especially the concentration of 3-hydroxybutyrate, acetone and succinate ${ }^{19}$ and 2-oxoglutarate and pseudouridine, ${ }^{51}$ was demonstrated. In another study CHF patients were characterized by higher levels of lactate, alanine, creatine, proline, isoleucine, leucine and lower levels of valine, glutamine, glutamate, choline, glycine, glucose, tyrosine and histidine ${ }^{50}$. More recently, metabolomic fingerprint of heart failure patients was characterized by lower levels of lactate, methionine and by higher levels of formate, phenylalanine, glucose, serine, acetate, hypoxantine, creatine and creatinine ${ }^{52}$. In the study by Lin et al ${ }^{53}$, HF metabolomic profile was characterized by higher levels of acetoacetate and urea and by lower levels of threonine, glycine, ethanol, histidine, alanine and tyrosine, consistent with our findings.

\section{The Effects of CRT on Systemic Metabolism}

Our study shows that CRT induces a significant shift in the metabolomic profile in HF patients, suggesting adaptive metabolic transitions. After CRT, an integral panel of the most important metabolites, including representatives of fatty acid (myristate, oleate, stearate, palmitate, palmitoleate, and heptadecanoate), amino acid (glutamine, glutamate, tryptophan, tyrosine, isoleucine, and $N$-methylhistidine), lipid (glycerol-1-phosphate and 2-HB), and Krebs cycle (citrate) metabolism, were improved. The improvement of substrate metabolism is indicated by changes in the succinate/glutamate and citrate/glutamate ratios; they are indicators of Krebs cycle activity, which are decreased in HF and significantly restored after CRT. Decreased levels of glutamine and malate and increased levels of citrate and alanine indicate a shift to more aerobic oxidation after CRT. A decreased glutamine/glutamate ratio in HF was also improved after CRT, indicating an improvement of ammonia fixation and detoxification. In addition, augmented levels of free fatty acids, amino acids, ketone bodies, and glycerol-1-phosphate suggest increased substrate reserve and shuttling of redox equivalents to mitochondria. Moreover, CRT also significantly increased the glucose/ palmitate ratio, suggesting enhanced glycolytic metabolism. Several metabolites return to control values after CRT. However, CRT had also specific metabolic effects such as activation of substrate utilization, amino acid and energy metabolism. CRT also improves metabolomics environment which is associated with a delta change in LVEF, as a evidence of improved heart performance similar to other reports ${ }^{18,43,52,54}$. Although post-CRT metabolomic profile improved significantly, many metabolite levels have not been restored 
to the normal range, indicating existence of resynchronization independent metabolic disturbances associated with $\mathrm{HF}^{49}, 50,53$. Clinically we observe that the extent of LVEF improvement is variable individually, and that only approximate 10\% CRT recipients are super-responders with LVEF normalization.

We found that CRT improves mitochondrial function as manifested in increased succinate/ glutamate and citrate/glutamate ratios compared to HF group, consistent with other reports ${ }^{17,42}$ which found augmented ATP synthase activity and glutamate/malate and succinate oxidations in heart mitochondria. Other recent studies revealed remodeling of sarcomeric proteins, including a-actinin, in $\mathrm{HF}$ that was reversed after $\mathrm{CRT}^{43}$. Moreover, at systemic level CRT promoted oxidative metabolism in peripheral muscles because of improved heart function and circulation. ${ }^{55}$ In summary, CRT induces pathways of adaptive metabolic transitions in the HF metabolomic profile, thus facilitating myocardial function recovery.

\section{Metabolic Biomarker for Response to CRT}

Predictive analytics is emerging area in personalized medicine ${ }^{56,57}$. This study suggests a specific plasma metabolic signature for response to CRT. Responders had higher baseline levels of isoleucine, phenylalanine, leucine, glucose, and valine and lower glutamate, essential for protein synthesis and metabolic signaling ${ }^{58,59}$. These metabolites may represent a better metabolic reserve and a higher potential for metabolic recovery in CRT responders. Analysis of metabolomic traits following CRT revealed that responders had higher amino acid, phosphoric acid, urea and Krebs cycle metabolite levels along with 2hydroxypyridine, which derivatives have antioxidant properties, and oxalic acid, which may indicate higher intake and metabolism of vitamin C. Higher plasma levels of citrate, creatine and creatinine in nonresponders indicate energetic deficiency. Nonresponders had also negative change in ornithine and higher increase in porphine levels compared to responders. Ornithine is a metabolite of urea cycle and ammonia detoxification while porphine is a product of degradation of porphyrins in heme containing proteins, which have energetic significance. Other studies have demonstrated that myocardial fatty acid extraction and calculated ATP synthesis flux may serve as biomarkers in identifying nonresponders to $\mathrm{CRT}^{18}$. Adaptive metabolic remodeling after CRT may facilitate development of prognostic tests for identifying CRT responders in a larger patient population. This requires larger scale metabolomics studies using complementary technologies as limited scale metabolite profiling may not be sufficient to establish predictive tests ${ }^{52}$. Similarly to our data, other studies ${ }^{52}$ have demonstrated a different metabolomic profile after CRT, which was also different from the fingerprint of healthy controls. The levels of serum metabolites - tyrosine, lactate, proline and alanine were significantly higher after CRT when compared to baseline ${ }^{52}$. However, in this study, which used only ${ }^{1} \mathrm{H}$ NMR technique, accuracy of discrimination between responders and non-responders remained low $^{52}$. In our study we used wider range metabolomic profiling using GC-MS and ${ }^{1} \mathrm{H}$ NMR techniques.

\section{Conclusions}

Our study suggests that HF is associated with a significantly altered metabolomic profile. Elevated plasma and myocardial free fatty acids may have negative impact on glycolytic 
ATP/energy transfer mechanism thus perpetuating energy imbalances and electrical instability. CRT improves the plasma metabolomic profile, including metabolic pathways of amino acid and lipid metabolism, ammonia detoxification, and Krebs cycle. A predictive panel of metabolites has been established to identify CRT responders. The results indicate that CRT facilitates synchronized metabolic signaling associated with improved substrate oxidation and overall energy metabolism.

\title{
Supplementary Material
}

Refer to Web version on PubMed Central for supplementary material.

\section{Acknowledgments}

Funding Sources

The authors gratefully acknowledge support from the National Institutes of Health, Marriott Foundation, and Mayo Foundation.

\author{
Abbreviations \\ CRT cardiac resynchronization therapy \\ GC-MS gas chromatography-mass spectrometry \\ HF heart failure \\ LV left ventricular \\ LVEF left ventricular ejection fraction \\ NYHA New York Heart Association \\ NMR nuclear magnetic resonance \\ OPLS-DA orthogonal partial least squares discriminant analysis \\ VIP variable importance in the projection
}

\section{References}

1. Ingwall JS. Energy metabolism in heart failure and remodelling. Cardiovasc Res. 2009; 81:412-419. [PubMed: 18987051]

2. Osterholt M, Sen S, Dilsizian V, Taegtmeyer H. Targeted metabolic imaging to improve the management of heart disease. JACC Cardiovasc Imaging. 2012; 5:214-226. [PubMed: 22340831]

3. Carley AN, Taegtmeyer H, Lewandowski ED. Matrix revisited: Mechanisms linking energy substrate metabolism to the function of the heart. Circ Res. 2014; 114:717-729. [PubMed: 24526677]

4. Doenst T, Nguyen TD, Abel ED. Cardiac metabolism in heart failure: Implications beyond atp production. Circ Res. 2013; 113:709-724. [PubMed: 23989714]

5. Dzeja PP, Redfield MM, Burnett JC, Terzic A. Failing energetics in failing hearts. Curr Cardiol Rep. 2000; 2:212-217. [PubMed: 10980895]

6. Cha YM, Dzeja PP, Shen WK, Jahangir A, Hart CY, Terzic A, Redfield MM. Failing atrial myocardium: Energetic deficits accompany structural remodeling and electrical instability. Am J Physiol Heart Circ Physiol. 2003; 284:H1313-H1320. [PubMed: 12626329] 
7. Cho H, Barth AS, Tomaselli GF. Basic science of cardiac resynchronization therapy: Molecular and electrophysiological mechanisms. Circulation: Arrhythmia and Electrophysiology. 2012; 5:594603. [PubMed: 22715238]

8. Gupta A, Akki A, Wang Y, Leppo MK, Chacko VP, Foster DB, Caceres V, Shi S, Kirk JA, Su J, Lai S, Paolocci N, Steenbergen C, Gerstenblith G, Weiss RG. Creatine kinase-mediated improvement of function in failing mouse hearts provides causal evidence the failing heart is energy starved. J Clin Invest. 2012; 122:291-302. [PubMed: 22201686]

9. Ventura-Clapier R, Garnier A, Veksler V, Joubert F. Bioenergetics of the failing heart. Biochim Biophys Acta. 2011; 1813:1360-1372. [PubMed: 20869993]

10. Lopaschuk GD, Ussher JR, Folmes CD, Jaswal JS, Stanley WC. Myocardial fatty acid metabolism in health and disease. Physiol Rev. 2010; 90:207-258. [PubMed: 20086077]

11. Dzeja PP, Hoyer K, Tian R, Zhang S, Nemutlu E, Spindler M, Ingwall JS. Rearrangement of energetic and substrate utilization networks compensate for chronic myocardial creatine kinase deficiency. J Physiol. 2011; 589:5193-5211. [PubMed: 21878522]

12. Bleeker GB, Bax JJ, Steendijk P, Schalij MJ, van der Wall EE. Left ventricular dyssynchrony in patients with heart failure: Pathophysiology, diagnosis and treatment. Nat Clin Pract Cardiovasc Med. 2006; 3:213-219. [PubMed: 16568130]

13. Cha YM, Rea RF, Wang M, Shen WK, Asirvatham SJ, Friedman PA, Munger TM, Espinosa RE, Hodge DO, Hayes DL, Redfield MM. Response to cardiac resynchronization therapy predicts survival in heart failure: A single-center experience. J Cardiovasc Electrophysiol. 2007; 18:10151019. [PubMed: 17711439]

14. Braunschweig F, Sorensen J, von Bibra H, Olsson A, Ryden L, Langstrom B, Linde C. Effects of biventricular pacing on myocardial blood flow and oxygen consumption using carbon-11 acetate positron emission tomography in patients with heart failure. Am J Cardiol. 2003; 92:95-99. [PubMed: 12842260]

15. Lindner O, Vogt J, Kammeier A, Wielepp P, Holzinger J, Baller D, Lamp B, Hansky B, Korfer R, Horstkotte D, Burchert W. Effect of cardiac resynchronization therapy on global and regional oxygen consumption and myocardial blood flow in patients with non-ischaemic and ischaemic cardiomyopathy. Eur Heart J. 2005; 26:70-76. [PubMed: 15615802]

16. Wang SB, Foster DB, Rucker J, O'Rourke B, Kass DA, Van Eyk JE. Redox regulation of mitochondrial atp synthase: Implications for cardiac resynchronization therapy. Circ Res. 2011; 109:750-757. [PubMed: 21817160]

17. Agnetti G, Kaludercic N, Kane LA, Elliott ST, Guo Y, Chakir K, Samantapudi D, Paolocci N, Tomaselli GF, Kass DA, Van Eyk JE. Modulation of mitochondrial proteome and improved mitochondrial function by biventricular pacing of dyssynchronous failing hearts. Circ Cardiovasc Genet. 2010; 3:78-87. [PubMed: 20160199]

18. Obrzut S, Tiongson J, Jamshidi N, Phan H, Hoh C, Birgersdotter-Green U. Assessment of metabolic phenotypes in patients with non-ischemic dilated cardiomyopathy undergoing cardiac resynchronization therapy. J. of Cardiovasc. Trans. Res. 2010; 3:643-651.

19. Du Z, Shen A, Huang Y, Su L, Lai W, Wang P, Xie Z, Xie Z, Zeng Q, Ren H, Xu D. 1h-nmr-based metabolic analysis of human serum reveals novel markers of myocardial energy expenditure in heart failure patients. PLoS One. 2014; 9:1-10.

20. Nemutlu E, Zhang S, Gupta A, Juranic NO, Macura SI, Terzic A, Jahangir A, Dzeja P. Dynamic phosphometabolomic profiling of human tissues and transgenic models by 18o-assisted (3)(1)p nmr and mass spectrometry. Physiol Genomics. 2012; 44:386-402. [PubMed: 22234996]

21. Trushina E, Nemutlu E, Zhang S, Christensen T, Camp J, Mesa J, Siddiqui A, Tamura Y, Sesaki H, Wengenack TM, Dzeja PP, Poduslo JF. Defects in mitochondrial dynamics and metabolomic signatures of evolving energetic stress in mouse models of familial alzheimer's disease. PLoS One. 2012; 7:e32737. [PubMed: 22393443]

22. Turer AT, Stevens RD, Bain JR, Muehlbauer MJ, van der Westhuizen J, Mathew JP, Schwinn DA, Glower DD, Newgard CB, Podgoreanu MV. Metabolomic profiling reveals distinct patterns of myocardial substrate use in humans with coronary artery disease or left ventricular dysfunction during surgical ischemia/reperfusion. Circulation. 2009; 119:1736-1746. [PubMed: 19307475] 
23. Cheng S, Rhee EP, Larson MG, Lewis GD, McCabe EL, Shen D, Palma MJ, Roberts LD, Dejam A, Souza AL, Deik AA, Magnusson M, Fox CS, O'Donnell CJ, Vasan RS, Melander O, Clish CB, Gerszten RE, Wang TJ. Metabolite profiling identifies pathways associated with metabolic risk in humans. Circulation. 2012; 125:2222-2231. [PubMed: 22496159]

24. Griffin JL, Atherton H, Shockcor J, Atzori L. Metabolomics as a tool for cardiac research. Nat Rev Cardiol. 2011; 8:630-643. [PubMed: 21931361]

25. Kang SM, Park JC, Shin MJ, Lee H, Oh J, Ryu do H, Hwang GS, Chung JH. (1)h nuclear magnetic resonance based metabolic urinary profiling of patients with ischemic heart failure. Clin Biochem. 2011; 44:293-299. [PubMed: 21167146]

26. Mercuro G, Bassareo PP, Deidda M, Cadeddu C, Barberini L, Atzori L. Metabolomics: A new era in cardiology? J Cardiovasc Med (Hagerstown). 2011; 12:800-805. [PubMed: 21934525]

27. Sabatine MS, Liu E, Morrow DA, Heller E, McCarroll R, Wiegand R, Berriz GF, Roth FP, Gerszten RE. Metabolomic identification of novel biomarkers of myocardial ischemia. Circulation. 2005; 112:3868-3875. [PubMed: 16344383]

28. Steffens DC, Wei J, Krishnan KR, Karoly ED, Mitchell MW, O'Connor CM, Kaddurah-Daouk R. Metabolomic differences in heart failure patients with and without major depression. J Geriatr Psychiatry Neurol. 2010; 23:138-146. [PubMed: 20101071]

29. Dunn WB, Broadhurst D, Begley P, Zelena E, Francis-McIntyre S, Anderson N, Brown M, Knowles JD, Halsall A, Haselden JN, Nicholls AW, Wilson ID, Kell DB, Goodacre R. Procedures for large-scale metabolic profiling of serum and plasma using gas chromatography and liquid chromatography coupled to mass spectrometry. Nat Protoc. 2011; 6:1060-1083. [PubMed: 21720319]

30. Alexander D, Lombardi R, Rodriguez G, Mitchell MM, Marian AJ. Metabolomic distinction and insights into the pathogenesis of human primary dilated cardiomyopathy. Eur J Clin Invest. 2011; 41:527-538. [PubMed: 21155767]

31. De Souza AI, Cardin S, Wait R, Chung YL, Vijayakumar M, Maguy A, Camm AJ, Nattel S. Proteomic and metabolomic analysis of atrial profibrillatory remodelling in congestive heart failure. J Mol Cell Cardiol. 2010; 49:851-863. [PubMed: 20655923]

32. Tracy CM, Epstein AE, Darbar D, Dimarco JP, Dunbar SB, Estes NA 3rd, Ferguson TB Jr, Hammill SC, Karasik PE, Link MS, Marine JE, Schoenfeld MH, Shanker AJ, Silka MJ, Stevenson LW, Stevenson WG, Varosy PD. 2012 accf/aha/hrs focused update of the 2008 guidelines for device-based therapy of cardiac rhythm abnormalities: A report of the american college of cardiology foundation/american heart association task force on practice guidelines. Heart Rhythm. 2012; 9:1737-1753. [PubMed: 22975672]

33. A J, Trygg J, Gullberg J, Johansson AI, Jonsson P, Antti H, Marklund SL, Moritz T. Extraction and $\mathrm{gc} / \mathrm{ms}$ analysis of the human blood plasma metabolome. Anal Chem. 2005; 77:8086-8094. [PubMed: 16351159]

34. Kind T, Wohlgemuth G, Lee do Y, Lu Y, Palazoglu M, Shahbaz S, Fiehn O. Fiehnlib: Mass spectral and retention index libraries for metabolomics based on quadrupole and time-of-flight gas chromatography/mass spectrometry. Anal Chem. 2009; 81:10038-10048. [PubMed: 19928838]

35. Nicholson JK, Foxall PJ, Spraul M, Farrant RD, Lindon JC. $750 \mathrm{mhz} 1 \mathrm{~h}$ and $1 \mathrm{~h}-13 \mathrm{c} \mathrm{nmr}$ spectroscopy of human blood plasma. Anal Chem. 1995; 67:793-811. [PubMed: 7762816]

36. Wishart DS, Lewis MJ, Morrissey JA, Flegel MD, Jeroncic K, Xiong Y, Cheng D, Eisner R, Gautam B, Tzur D, Sawhney S, Bamforth F, Greiner R, Li L. The human cerebrospinal fluid metabolome. J Chromatogr B Analyt Technol Biomed Life Sci. 2008; 871:164-173.

37. Stein SE. An integrated method for spectrum extraction and compound identification from gas chromatography/mass spectrometry data. J Am Soc Mass Spectrom. 1999; 10:770-781.

38. Styczynski MP, Moxley JF, Tong LV, Walther JL, Jensen KL, Stephanopoulos GN. Systematic identification of conserved metabolites in $\mathrm{gc} / \mathrm{ms}$ data for metabolomics and biomarker discovery. Anal Chem. 2007; 79:966-973. [PubMed: 17263323]

39. Elrod JW, Wong R, Mishra S, Vagnozzi RJ, Sakthievel B, Goonasekera SA, Karch J, Gabel S, Farber J, Force T, Brown JH, Murphy E, Molkentin JD. Cyclophilin d controls mitochondrial pore-dependent $\mathrm{ca}(2+)$ exchange, metabolic flexibility, and propensity for heart failure in mice. $\mathbf{J}$ Clin Invest. 2010; 120:3680-3687. [PubMed: 20890047] 
40. Abraham WT, Hayes DL. Cardiac resynchronization therapy for heart failure. Circulation. 2003; 108:2596-2603. [PubMed: 14638522]

41. Barth A, Chakir K, Kass D, Tomaselli G. Transcriptome, proteome, and metabolome in dyssynchronous heart failure and crt. J. of Cardiovasc. Trans. Res. 2012; 5:180-187.

42. Kirk JA, Kass DA. Electromechanical dyssynchrony and resynchronization of the failing heart. Circ Res. 2013; 113:765-776. [PubMed: 23989718]

43. Lichter JG, Carruth E, Mitchell C, Barth AS, Aiba T, Kass DA, Tomaselli GF, Bridge JH, Sachse FB. Remodeling of the sarcomeric cytoskeleton in cardiac ventricular myocytes during heart failure and after cardiac resynchronization therapy. J Mol Cell Cardiol. 2014; 72:186-195. [PubMed: 24657727]

44. Ukkonen H, Beanlands RS, Burwash IG, de Kemp RA, Nahmias C, Fallen E, Hill MR, Tang AS. Effect of cardiac resynchronization on myocardial efficiency and regional oxidative metabolism. Circulation. 2003; 107:28-31. [PubMed: 12515738]

45. Pietersen HG, Langenberg CJ, Geskes G, Soeters PB, Wagenmakers AJ. Glutamate metabolism of the heart during coronary artery bypass grafting. Clin Nutr. 1998; 17:73-75. [PubMed: 10205320]

46. Ardehali H, Sabbah HN, Burke MA, Sarma S, Liu PP, Cleland JG, Maggioni A, Fonarow GC, Abel ED, Campia U, Gheorghiade M. Targeting myocardial substrate metabolism in heart failure: Potential for new therapies. Eur J Heart Fail. 2012; 14:120-129. [PubMed: 22253453]

47. Leyva F, Anker S, Swan JW, Godsland IF, Wingrove CS, Chua TP, Stevenson JC, Coats AJ. Serum uric acid as an index of impaired oxidative metabolism in chronic heart failure. Eur Heart J. 1997; 18:858-865. [PubMed: 9152657]

48. Marazzi G, Rosanio S, Caminiti G, Dioguardi FS, Mercuro G. The role of amino acids in the modulation of cardiac metabolism during ischemia and heart failure. Curr Pharm Des. 2008; 14:2592-2604. [PubMed: 18991676]

49. Tenori L, Hu X, Pantaleo P, Alterini B, Castelli G, Olivotto I, Bertini I, Luchinat C, Gensini GF. Metabolomic fingerprint of heart failure in humans: A nuclear magnetic resonance spectroscopy analysis. International Journal of Cardiology. 2013; 168:e113-e115. [PubMed: 24007967]

50. Wang J, Li Z, Chen J, Zhao H, Luo L, Chen C, Xu X, Zhang W, Gao K, Li B, Zhang J, Wang W. Metabolomic identification of diagnostic plasma biomarkers in humans with chronic heart failure. Mol Biosyst. 2013; 9:2618-2626. [PubMed: 23959290]

51. Dunn WB, Broadhurst DI, Deepak SM, Buch MH, McDowell G. Serum metabolomics reveals many novel metabolic markers of heart failure, including pseudouridine and 2-oxoglutarate. Metabolomics. 2007; 3:413-426.

52. Padeletti L, Modesti PA, Cartei S, Checchi L, Ricciardi G, Pieragnolia P, Sacchi S, Padeletti M, Alterini B, Pantaleo P, Hu X, Tenori L, Luchinat C. Metabolomic does not predict response to cardiac resynchronization therapy in patients with heart failure. J Cardiovasc Med (Hagerstown). 2014; 15:295-300. [PubMed: 24699011]

53. Lin D, Hollander Z, Meredith A, Stadnick E, Sasaki M, Cohen Freue G, Qasimi P, Mui A, Ng RT, Balshaw R, Wilson-McManus JE, Wishart D, Hau D, Keown PA, McMaster R, McManus BM. Molecular signatures of end-stage heart failure. Journal of Cardiac Failure. 2011; 17:867-874. [PubMed: 21962426]

54. Kirk JA, Holewinski RJ, Kooij V, Agnetti G, Tunin RS, Witayavanitkul N, de Tombe PP, Gao WD, Van Eyk J, Kass DA. Cardiac resynchronization sensitizes the sarcomere to calcium by reactivating gsk-33. J Clin Invest. 2014; 124:129-139. [PubMed: 24292707]

55. Jaussaud J, Blanc P, Bordachar P, Roudaut R, Douard H. Response to cardiac resynchronization therapy: The muscular metabolic pathway. Cardiol Res Pract. 2011; 2011:830279. [PubMed: 21197405]

56. Chen R, Mias GI, Li-Pook-Than J, Jiang L, Lam HY, Miriami E, Karczewski KJ, Hariharan M, Dewey FE, Cheng Y, Clark MJ, Im H, Habegger L, Balasubramanian S, O'Huallachain M, Dudley JT, Hillenmeyer S, Haraksingh R, Sharon D, Euskirchen G, Lacroute P, Bettinger K, Boyle AP, Kasowski M, Grubert F, Seki S, Garcia M, Whirl-Carrillo M, Gallardo M, Blasco MA, Greenberg PL, Snyder P, Klein TE, Altman RB, Butte AJ, Ashley EA, Gerstein M, Nadeau KC, Tang H, Snyder M. Personal omics profiling reveals dynamic molecular and medical phenotypes. Cell. 2012; 148:1293-1307. [PubMed: 22424236] 
57. Erogbogbo F, May J, Swihart M, Prasad PN, Smart K, Jack SE, Korcyk D, Webster M, Stewart R, Zeng I, Zullig M, Bakeev K, Jamieson M, Kasabov N, Gopalan B, Liang L, Hu R, Schliebs S, Villas-Boas S, Gladding P. Bioengineering silicon quantum dot theranostics using a network analysis of metabolomic and proteomic data in cardiac ischemia. Theranostics. 2013; 3:719-728. [PubMed: 24019856]

58. Witham WG, Yester KA, McGaffin KR. A high leucine diet mitigates cardiac injury and improves survival after acute myocardial infarction. Metabolism. 2013; 62:290-302. [PubMed: 22935555]

59. Huang Y, Zhou M, Sun H, Wang Y. Branched-chain amino acid metabolism in heart disease: An epiphenomenon or a real culprit? Cardiovasc Res. 2011; 90:220-223. [PubMed: 21502372] 


\section{A Discrimination of metabolomic profiles}
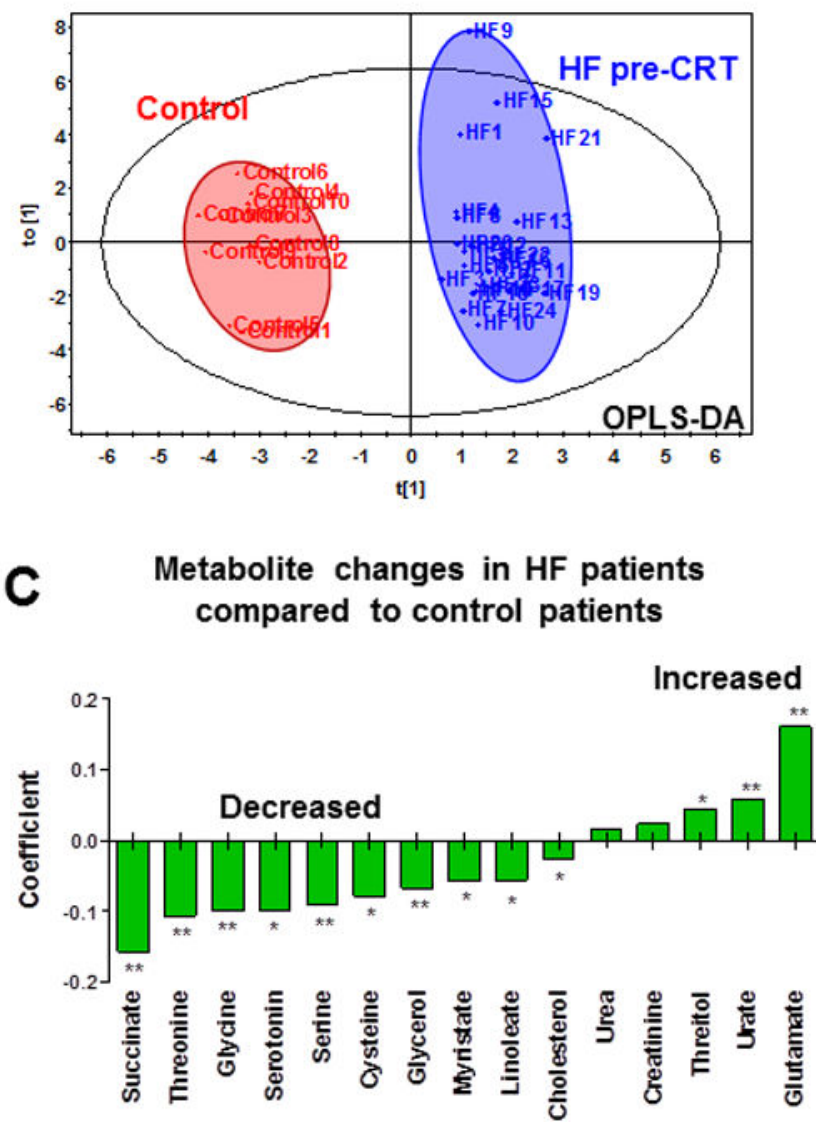
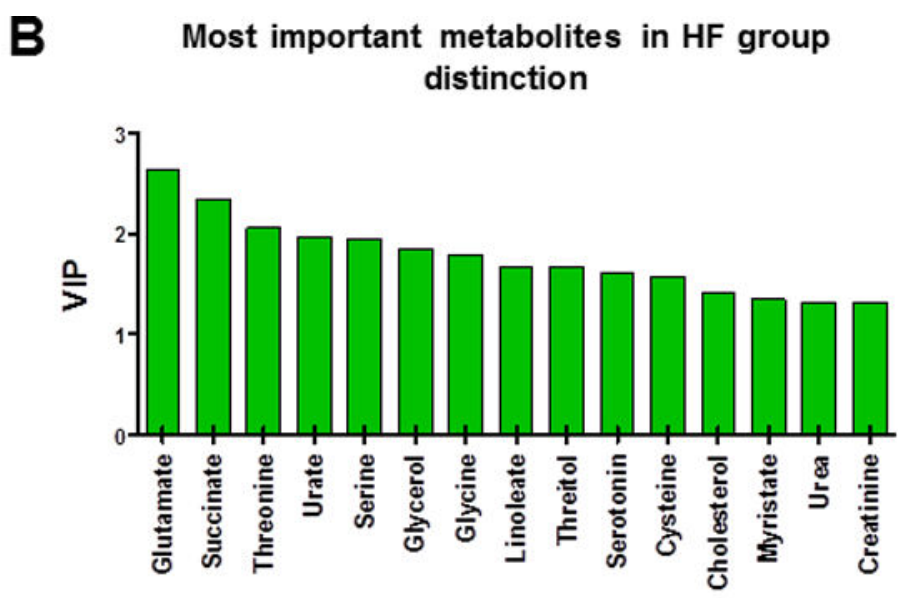

Detabolic pathways affected in HF

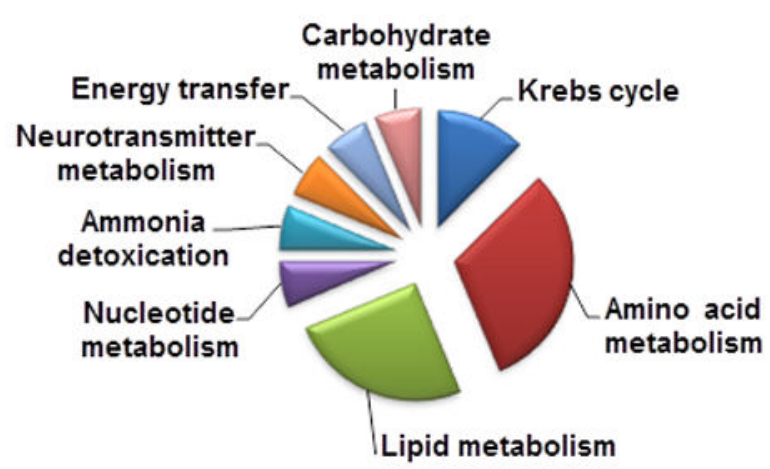

Figure 1.

Metabolomic signature of heart failure (HF). A, Changes in plasma metabolomic profile of HF patients by orthogonal partial least squares discriminant analysis (OPLS-DA). B, Most important metabolites in the metabolomic signature of HF by variable importance in the projection (VIP). C, Pattern of plasma metabolite changes in HF patients $(\mathrm{N}=24, * P<.05$; ** $P<.001)$. D, Metabolic pathways most affected in HF as deduced from an altered plasma metabolomic profile. 


\section{A Discrimination of metabolomic profiles}

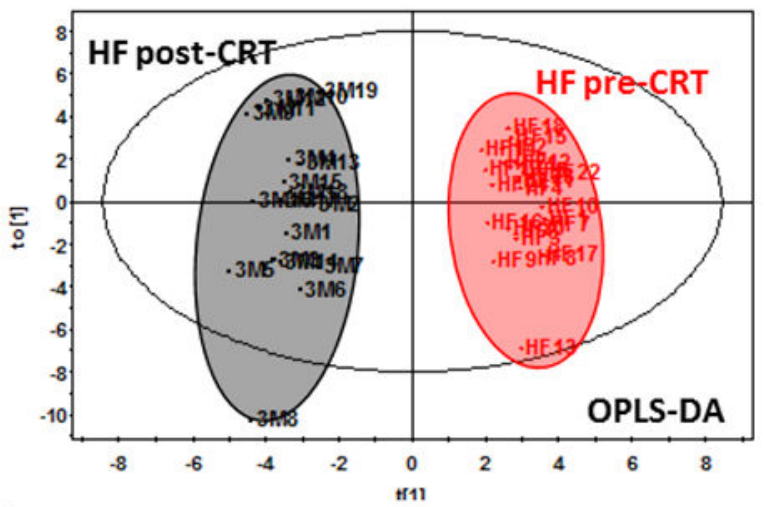

C

Figure 2. plasma metabolomic profile.
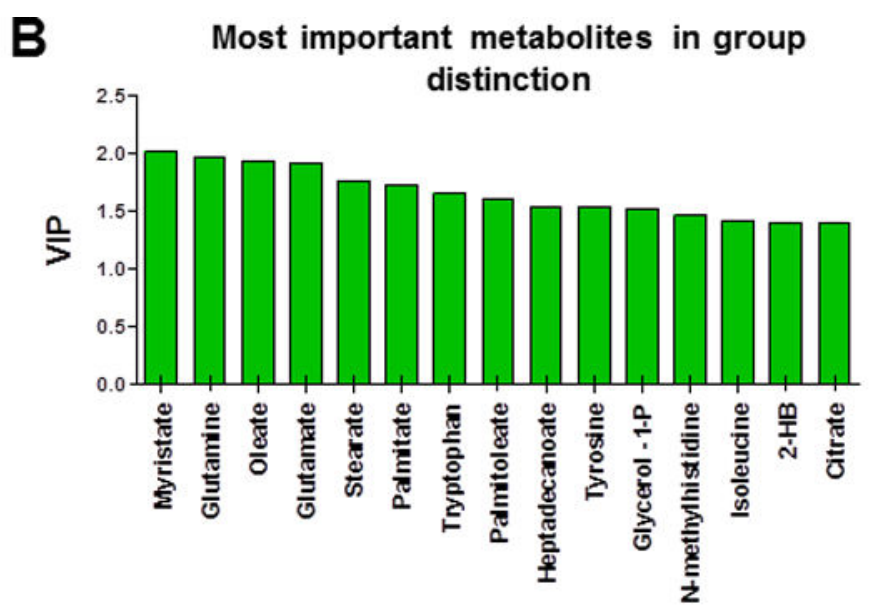

D Metabolic pathways most affected after CRT

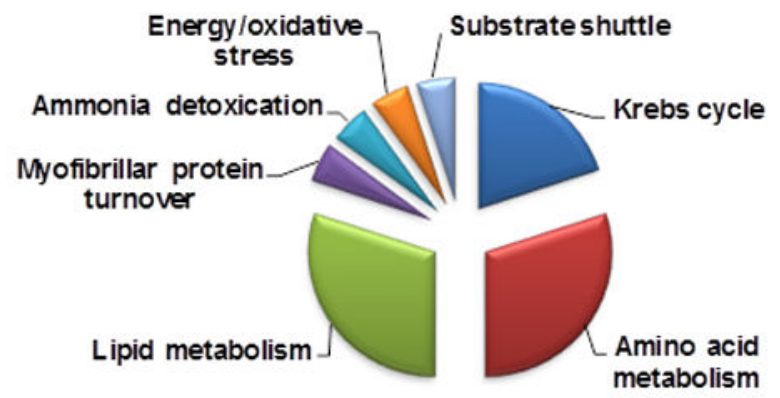

Metabolomic signature of cardiac resynchronization therapy (CRT). A, Changes in plasma metabolomic profiles of patients with heart failure (HF) after CRT by orthogonal partial least squares discriminant analysis (OPLS-DA). 3M indicates 3-month follow-up. B, Most important metabolites in metabolomic signature of CRT by variable importance in the projection (VIP). C, Pattern of plasma metabolite changes in HF patients after CRT (N=19, ** $P<.001)$. D, Metabolic pathways most affected after CRT as deduced from an altered 

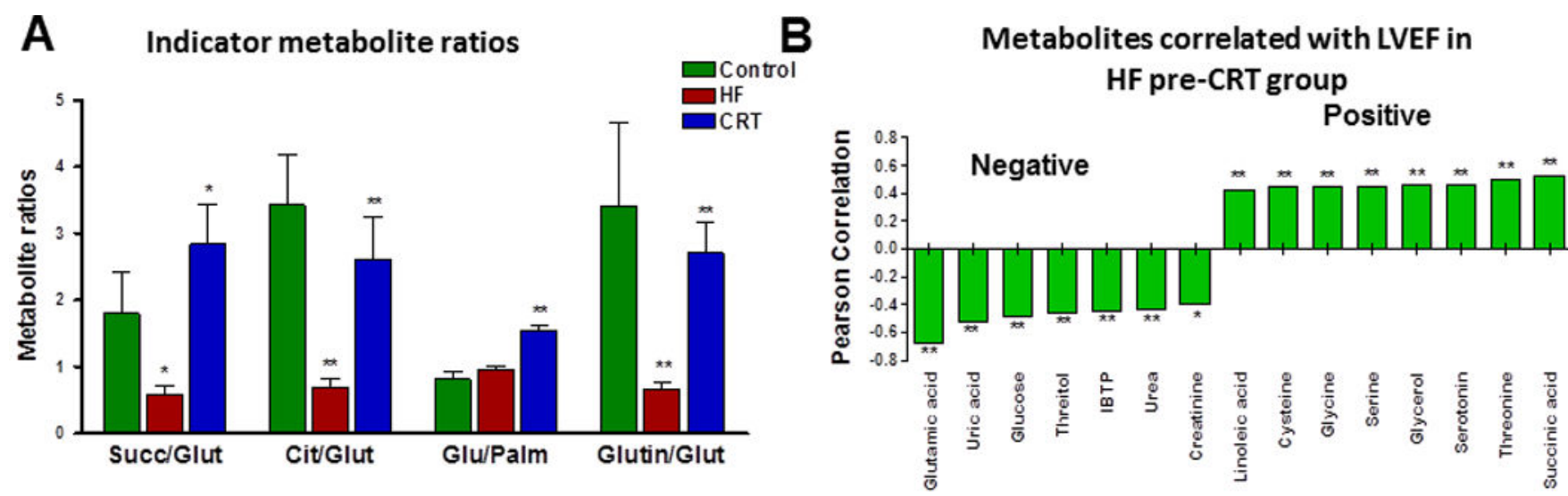

\section{Metabolites correlated with LVEF in HF post-CRT group}

Positive

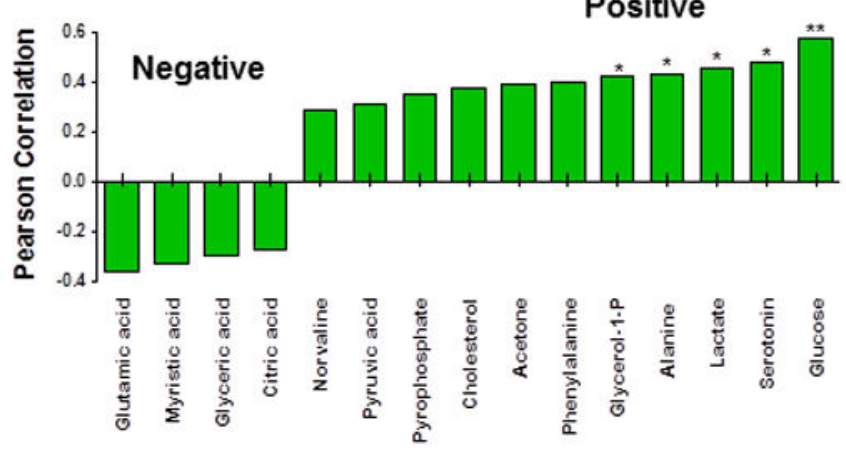

D

Metabolites correlated with heart delta LVEF in HF post-CRT group

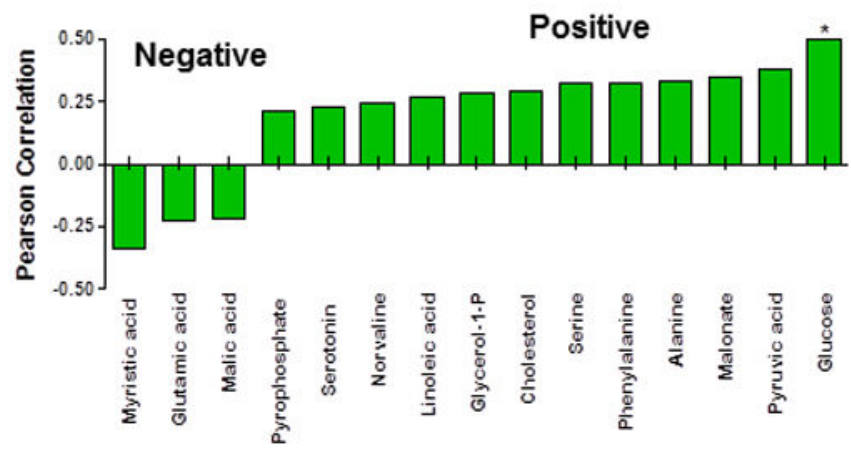

Figure 3.

Improvement of indicator metabolite ratios and correlations between plasma metabolites and cardiac performance (left ventricle ejection fraction [LVEF]) after cardiac resynchronization therapy (CRT). A, Changes in indicator metabolite ratios in HF pre-CRT and HF post-CRT. $\mathrm{B}$, A panel of metabolites that correlate positively and negatively with LVEF in HF pre-CRT group (*P<.05; ** $P<.001)$. C, A panel of metabolites that correlate positively and negatively with LVEF in HF post-CRT group $(* P<.05$; $* * P<.001)$. D, A panel of metabolites that correlate positively and negatively with delta LVEF in HF post-CRT group ( $\left.{ }^{*} P<.05\right)$. Cit/Glut indicates citrate/glutamate; Glu/Palm, glucose/palmitate; Glutin/Glut, glutamine/glutamate; HF, heart failure; Succ/Glut, succinate/glutamate. 
A Discrimination of HF pre-CRT metabolomic profiles

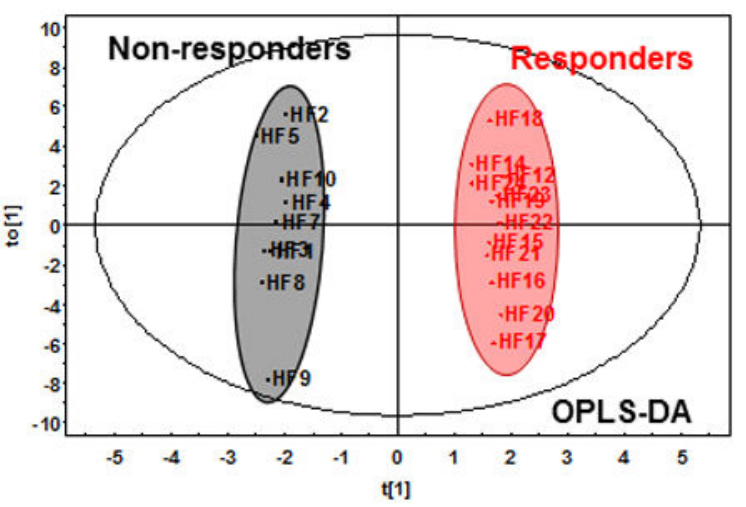

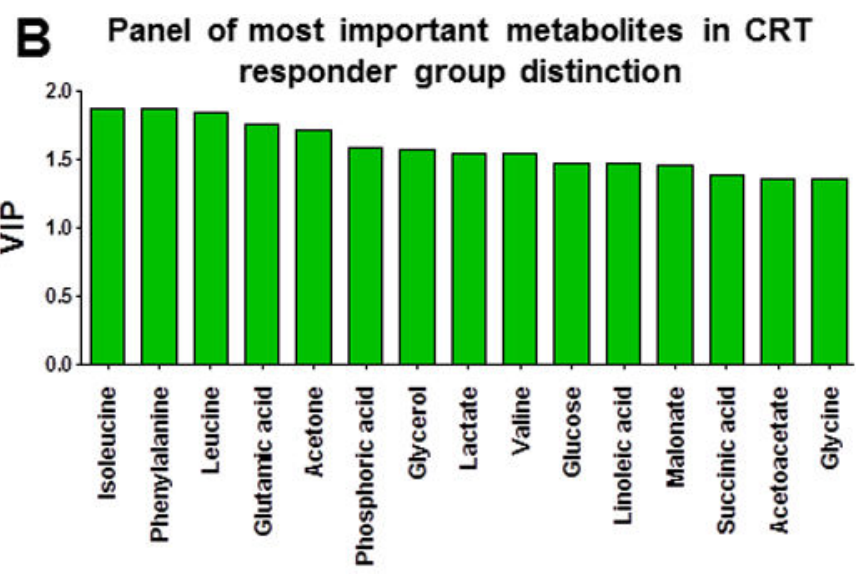

C

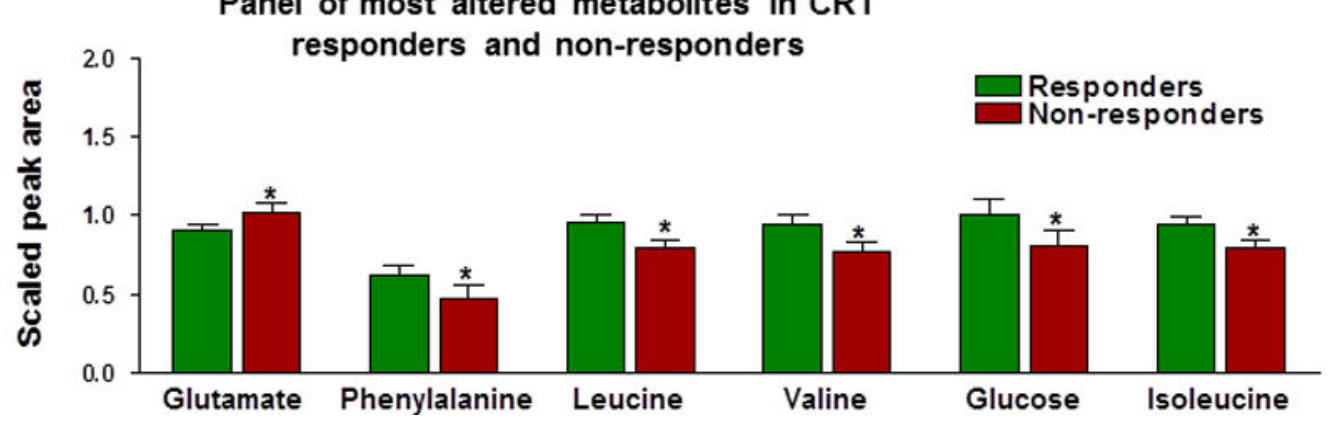

Figure 4.

Metabolomic profiles of 9 responders and 13 nonresponders to cardiac resynchronization therapy (CRT). A, Distinction of basal plasma metabolomic profiles of responders and nonresponders to CRT by orthogonal partial least squares discriminant analysis (OPLS-DA). $\mathrm{B}$, An integral panel of the most important metabolites permitting prediction of responders by variable importance in the projection (VIP). C, A panel of the most important metabolite differences between responders and nonresponders ( $\left.{ }^{*} P<.05\right)$. 


\section{Table 1}

Baseline Characteristics of the HF and Control Patients*

\begin{tabular}{|l|l|l|l|}
\hline Variable & $\begin{array}{l}\text { HF Patients } \\
(\mathbf{N = 2 4 )}\end{array}$ & $\begin{array}{l}\text { Control } \\
\text { Patients } \\
(\mathbf{N = 1 0})\end{array}$ & P Value \\
\hline Age, y & $65.3 \pm 15.0$ & $65.4 \pm 13.0$ & .79 \\
\hline Male sex, & $20(83)$ & $6(60)$ & .19 \\
\hline CAD & $11(46)$ & $1(10)$ & .06 \\
\hline Ischemic cardiomyopathy & $10(42)$ & 0 & .02 \\
\hline Hypertension & $11(46)$ & $3(30)$ & .46 \\
\hline Diabetes & $9(38)$ & $1(10)$ & .21 \\
\hline QRS duration, ms & $172 \pm 24$ & $103 \pm 23$ & $<.001$ \\
\hline LVEF, \% & $23.5 \pm 6.3$ & $62.9 \pm 3.3$ & $<.001$ \\
\hline LVEDD, mm & $66.6 \pm 9.9$ & $48.6 \pm 2.7$ & $<.001$ \\
\hline ACEI/ARB & $16(67)$ & 0 & $<.001$ \\
\hline$\beta$-Blocker & $18(75)$ & $5(50)$ & .23 \\
\hline Digoxin & $7(29)$ & 0 & .08 \\
\hline Statin & $14(58)$ & 0 & .002 \\
\hline
\end{tabular}

Abbreviations: ACEI/ARB, angiotensin-converting enzyme inhibitor or angiotensin receptor blocker; CAD, coronary artery disease; LVEDD, left ventricular end-diastolic dimension; LVEF, left ventricular ejection fraction.

Values are number (percentage) or mean \pm standard error of the mean unless indicated otherwise. 
Table 2

Comparison of Clinical Parameters Before and After CRT*

\begin{tabular}{|l|l|l|l|l|}
\hline Variable & $\begin{array}{l}\text { Control } \\
\text { N=10 }\end{array}$ & $\begin{array}{l}\text { Pre-CRT } \\
\text { N=24 }\end{array}$ & $\begin{array}{l}\text { Post-CRT } \\
\text { N=24 }\end{array}$ & $\begin{array}{l}\text { P Value } \\
\text { Pre vs. Post }\end{array}$ \\
\hline NYHA class, grade & $1.0 \pm 0$ & $2.7 \pm 0.4$ & $2.1 \pm 0.6$ & $<.03$ \\
\hline LVEF, \% & $62.9 \pm 3.3$ & $23.5 \pm 6.3$ & $32.0 \pm 8.6$ & $<.001$ \\
\hline LVEDD, mm & $48.6 \pm 2.7$ & $66.6 \pm 9.9$ & $64.9 \pm 10.6$ & .15 \\
\hline Mitral regurgitation, grade & $1.7 \pm 0.6$ & $2.3 \pm 0.6$ & $2.0 \pm 0.6$ & $<.01$ \\
\hline Pulmonary artery systolic pressure, $\mathrm{mm} \mathrm{Hg}$ & $30.6 \pm 4.7$ & $43.02 \pm 12.6$ & $38.2 \pm 11.6$ & $<.01$ \\
\hline
\end{tabular}

Abbreviations: LVEDD, left ventricular end-diastolic dimension; LVEF, left ventricular ejection fraction; NYHA, New York Heart Association.

* Values are mean \pm standard error of the mean unless indicated otherwise. 